\section{Visual inhomogeneity and eye movements in multistable perception}

\section{MIGUEL A. GARCIAA-PÉREZ \\ Universidad Complutense, Campus Somosaguas Madrid, Spain}

For a long time figural multistability has attracted the attention of psychologists, because to understand how this phenomenon occurs might help one to understand perceptual processing in general. Figural multistability appears to be a necessary consequence of the normal functioning of our visual system, and hence it is something that any theory of visual processing should be able to account for with recourse to the mechanisms that explain normal, or correct, perception.

This interest has generated much research aimed at determining the factors that control the alternation in interpretations inherent in the phenomenon of perceptual multistability. The roles of eye movements, type of viewing (either binocular or dichoptic), stimulus contrast, state of light-dark adaptation, focus, mean luminance, and many other factors have been investigated, but the results of these research efforts have yielded no conclusive evidence in favor of any of these factors' being the cause of alternations. At best, these studies have proved the existence of some empirical regularities, but they have not offered any explanation for why these regularities should hold; they have failed to provide a deductive link between the basic properties of the visual system and the empirical results.

Among the factors shown to be involved, eye movements have seemed to be the more likely cause of the alternations, but eye-movement recordings made of subjects inspecting ambiguous figures have not consistently revealed that a change in fixation either follows or precedes an alternation (see Gale \& Findlay, 1983). The fact that reversals may also occur voluntarily (see Liebert \& Burk, 1985 ) adds a further complication, because it clearly reveals that both top-down and bottom-up processes are involved. Consequently, it is difficult to trace the boundary between what perceptual multistability owes to early visual processing, and what cognitive processing adds to it. It is the aim of this note to show how visual inhomogeneity, a well-known and well-established basic property of early visual processing, may be responsible for some of the alternations that occur when the eyes move across a multistable figure.

This work has been supported with grants from the Universidad Complutense and the Fundación Ramón Areces for a research project entitled Visión Artificial y Visión Humana: Aplicaciones a la Percepción Visual en Roborica. Address correspondence to Miguel A. Garcia-Pérez, Laboratorio de Visión, Facultad de Psicología, Universidad Complutense, Campus de Somosaguas, 28023 Madrid, Spain.
To accomplish this, a few words are necessary about the currently accepted explanation of alternations in multistable perception. Apart from minor effects of varying experimental conditions on the rate of alternations, it is now widely accepted that the alternations themselves are due to shifts of attention to specific areas in the figure that differentially tend to evoke one interpretation or the other (Gale \& Findlay, 1983; Kawabata, 1986; Tsal \& Kolbet, 1985). This statement is based on the fact that fixation at some areas consistently gives rise to one interpretation, whereas fixation at other areas tends to elicit the other. Nonetheless, in some studies (e.g., Goolkasian, 1987) this correlation has not been found. The present note provides a basis for explaining the discrepancy.

It is important to realize that there are two unresolved issues in the above statement about the role of attentional shifts in alternations. The first one is the twofold question of why a shift of attention is sometimes associated with a change in the fixation point, and how movements of the eye affect the reversal process. The second is the question of why different features of the picture are more likely to evoke one interpretation than the other. This second question will not be addressed here, because it raises semantic issues that fall outside the context of early visual processing. In response to the first issue, it will be argued here that perceptually available detail information is essentially what determines the immediate interpretation of a multistable figure, and that eye movements across large figures help accomplish attentional shifts by changing the detail information that is available. It will also be shown that both factors may explain the seemingly contradictory results regarding the role of eye movements in alternations.

When we fixate a location in a scene, visual inhomogeneity prevents the perceived image from having equal resolution throughout. Thus, we see the outside world through a window of varying resolution such that fine detail is available only within a small area around the fixation point. García-Perez (1988) has found that the radius of that area is less than $2^{\circ}$ under conditions of highcontrast stimulation, and that this radius gets larger as the spatial scale gets coarser (see García-Pérez, 1988, Figure 5). The consequences of visual inhomogeneity on the visibility of high spatial frequency information when one inspects ambiguous figures are illustrated below.

Figure 1a shows a digitized version of Boring's figure (actually originated by Hill, 1915). Figure 1b shows the image as it is perceived when the picture in Figure la subtends $15^{\circ} \times 20^{\circ}$ and the eyes are fixated midway between the features corresponding to the young woman's eye and ear. (This image has been obtained with a procedure that is fully described in García-Pérez, 1987, using the empirical data from García-Pérez, 1988, Table 2.) Figure kc shows the high spatial frequency (above $16 \mathrm{cpd}$ ) information that is available in these conditions. Note that these 


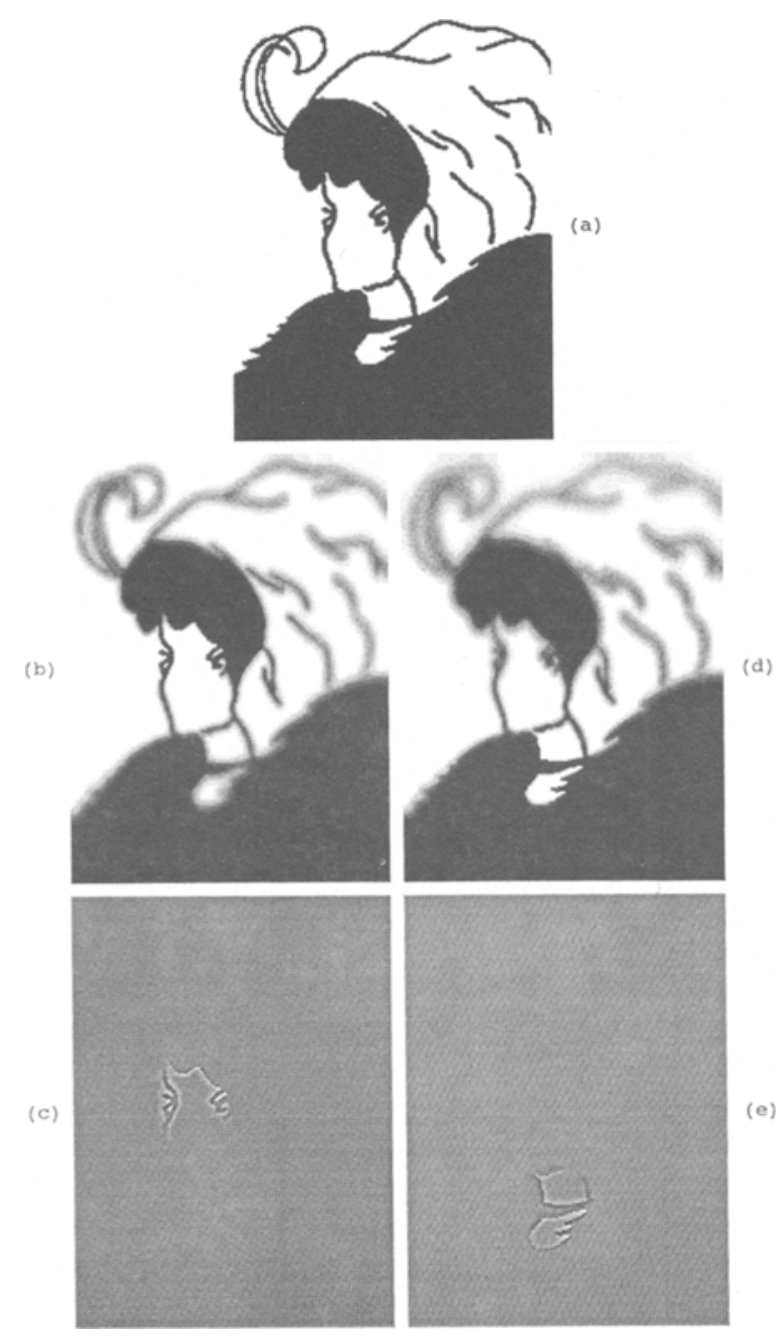

Figure 1. (a) Boring's young/old woman. (b) Perceived image when the eyes fixate midway between the young woman's eye and ear at a distance such that the image subtends $15^{\circ} \times 20^{\circ}$. (c) High spatial frequency (above $16 \mathrm{cpd}$ ) information available under such circumstances. (d) Perceived image when the fixation point is on the old woman's mouth under the same viewing conditions. (e) High spatial frequency information available in this case.

edges clearly outline the young woman's profile. This is in close agreement with the empirical fact that nearly $90 \%$ of the fixations around this position give rise to the young woman interpretation when the image of Figure 1a is viewed under these circumstances (Gale \& Findlay, 1983, Figure 3) and that subjects tend to fixate this area when they are instructed to concentrate on the young woman aspects (Gale \& Findlay, 1983). On the other hand, Figures $1 \mathrm{~d}$ and $1 \mathrm{e}$ are similar to Figures $1 \mathrm{~b}$ and $1 \mathrm{c}$, except that the fixation location has been changed to the mouth of the old woman. Clearly, the edges in Figure 1e do not induce the young woman interpretation. Actually, they enhance more the old woman interpretation, albeit not as clearly as the edges in Figure 1c do with regard to the young woman. This may explain why the percentage of old woman responses when this area is fixated un- der these conditions (slightly above $60 \%$; see Gale \& Findlay, 1983, Figure 3) is not as high as the corresponding percentage of young woman responses for the young woman area.

Then, if fine detail information, which is available from only a small area around the fixation position because of visual inhomogeneity, has the main role in object recognition, the above analysis should show why eye movements are sometimes necessary to produce an alternation in interpretation when large ambiguous figures are inspected: the features more likely to evoke the alternate interpretation fall outside the restricted field of view that is subserved by high-spatial-frequency visual channels. On the other hand, if the figure is small, then all biasing elements will be visible at a glance, and attention can be shifted from one to the other without the need for eye movements. Hence, the retinal size of an ambiguous picture is a major factor in determining whether eye movements and alternations will or will not be correlated. However, the angle subtended by ambiguous pictures has not been reported in all studies (e.g., Goolkasian, 1987; Wilton, 1979).

The above points are not restricted to perceptual multistability caused by ambiguous figures. Figure-ground reversals associated with eye movements can be explained similarly. Figure 2 shows an analogous analysis performed on a digitized version of Rubin's vase/face (Figure 2a). Figures $2 b$ and $2 d$ show, respectively, the perceived image when the picture subtends $9^{\circ} \times 12^{\circ}$ and either the center of the vase hollow or the right profile nose is fixated. Figures $2 \mathrm{c}$ and $2 \mathrm{e}$ show the available high spatial frequency edges in each case. It can easily be seen that fixation at the vase hollow makes the vase features more (alternatively, the profile features less) salient, whereas fixation near the human profiles biases edge information toward this interpretation.

Visual inhomogeneity can therefore be said to play a role in perceptual multistability. The preceding argument is based on the notion that detail information that remains available after the space-variant filtering performed by our visual system is primarily concerned in determining the interpretation of a figure. Image edges have been said to play a fundamental role in visual processing (Marr, 1982 ), and it has recently been shown (Biederman \& Ju, 1988) that surface characteristics (i.e., color, brightness, texture, etc.) are relevant to object recognition only to the extent that they contribute to the defining of image edges. It is true that edges can be found at a variety of spatial scales, but it is likely that high spatial frequency edges are mostly relevant to object recognition, since they provide the finest information about the image. This is in agreement with Biederman and Ju's (1988) results, since the outlined objects they used in their experiments had high spatial frequency information almost exclusively. Some authors have even discussed whether cells in striate cortex do detect edges or not (Hochstein \& Spitzer, 1984; Richter \& Ullman, 1986). The fact that visual inhomogeneity suppresses most of the detail information in 

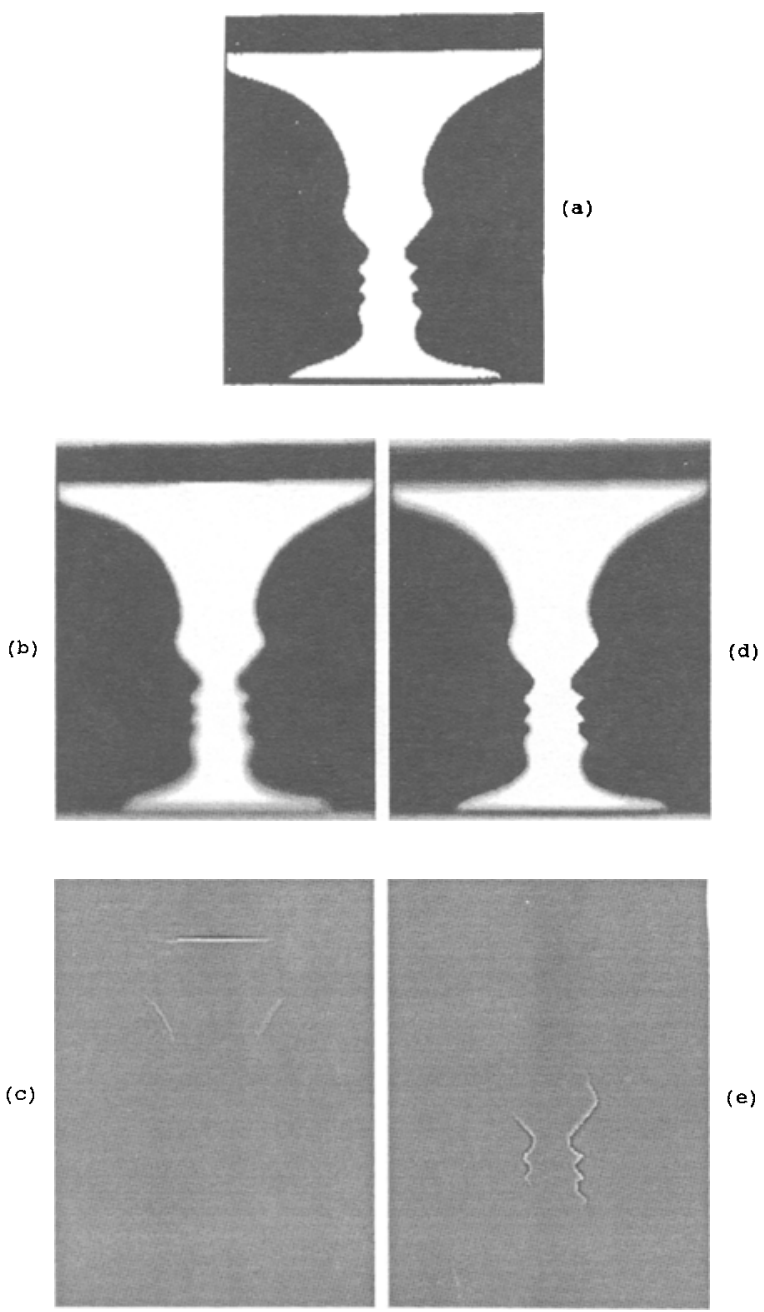

Figure 2. (a) Rubin's vase/face. (b) Perceived image when the eyes fixate on the center of the vase hollow at a distance such that the image subtends $9^{\circ} \times 12^{\circ}$. (c) High spatial frequency (above $16 \mathrm{cpd}$ ) information available under such circumstances. (d) Perceived image when the fixation point is on the nose of the right human profile under the same viewing conditions. (e) High spatial frequency information available in this case.

the visual field and that fixation location determines which detail information will still be available argues in favor of a meaningful role of space-variant visual processing in disambiguating multistable figures.

Previous attempts to explain perceptual multistability as a result of the filtering performed in early visual processing (e.g., Ginsburg, 1978) were seriously limited because the visual system was considered (wrongly) to be homogeneous: if a single filtered image exists, explaining why it evokes one interpretation sometimes and then the other seems unfeasible except with respect to figures containing objects of different sizes, which can be reversed by changing the spatial scale. Visual inhomogeneity, on the other hand, gives rise to as many filtered images as there are meaningfully different fixation locations, and the possibility exists of relating some of them to each possible interpretation of the figure.
This account of alternations applies to other types of multistable figures as well. Whenever image features can be found empirically to be more or less likely to evoke one interpretation or the other in a multistable figure, particular fixations on a large picture can be shown to produce selection and suppression of high spatial frequency edges in a manner consistent with the experimental results. This idea can easily be applied to the interpretation of Kawabata's (1986) results regarding Necker's cube, or the results obtained by Tsal and Kolbet (1985) with the duck/rabbit and bird/plane figures. The same considerations apply also to many other multistable figures, including Fisher's (1967) man/lady or rat/man, the Schröder staircase, and so forth, as well as to the effect of contextual information on the perceived pointing of ambiguous triangles (García-Pérez, 1989). Fixation at a given location will select detail information from that area only, thus facilitating the appropriate interpretation. In some sense, the differentially blurred image that results from visual inhomogeneity and a particular fixation location can be conceived as a biased version of the ambiguous distal figure, in much the same way as the biased drawings of the figures that are often produced for purposes of research. Still unresolved is the question of why particular features of the figure are more likely to evoke one interpretation than the other, but the answer to this question surely lies beyond early visual processing.

Regardless of the points made above, it is important to note that visual inhomogeneity in itself does not account for the alternations, nor does it explain perceptual multistability. It was said at the beginning of this note that alternations in interpretation do occur in the absence of eye movements (i.e., with a single retinal image), and it is also true that alternations can be controlled at will ( $\mathrm{Lie}$ bert \& Burk, 1985). Hence, many factors may be involved after early visual processing. What visual inhomogeneity seems to do is allow reversals to be easily achieved by selectively providing subsequent visual and cognitive processing with image data relevant to one or the other interpretation. Several models for multistable perception exist that aim at explaining this phenomenon from different standpoints (e.g., see Babich \& Standing, 1981; Gale \& Findlay, 1983; Ginsburg, 1978; Kawamoto \& Anderson, 1985; Kienker, Sejnowski, Hinton, \& Schumacher, 1986; Köhler \& Wallach, 1944; Long, Toppino, \& Kostenbauder, 1983; Poston \& Stewart, 1978; Robinson, 1972; Shafer, 1976). But, surprisingly, a well-established structural and functional characteristic (namely, visual inhomogeneity) of the system involved in such a phenomenon, and which makes possible any form of perception, has not been taken into account, nor even mentioned, in these proposed explanations. The demonstration provided here shows that visual inhomogeneity may explain some of the automatic changes of interpretation that are associated with eye movements, which possibly account for most of what the mechanisms of early visual processing have to do with perceptual multistability. Perhaps the rest of the story has to be explained in terms of the intervention of cognitive processes. 


\section{REFERENCES}

Babich, S., \& Standing, L. (1981). Satiation effects with reversible figures. Perceptual \& Motor Skills, 52, 203-210.

Biederman, I, Ju, G. (1988). Surface- versus edge-based determinants of visual recognition. Cognitive Psychology, 20, 38-64.

Fisher, G. H. (1967). Preparation of ambiguous stimulus materials. Perception \& Psychophysics, 2, 421-422.

GALE, A. G., FINDLAY, J. M. (1983). Eye movement patterns in viewing ambiguous figures. In R. Groner, C. Menz, D. F. Fisher, \& R. A. Monty (Eds.), Eye movements and psychological functions: International views (pp. 145-168). Hillsdale, NJ: Erlbaum.

García-Pérez, M. A. (1987). An efficient approach to modeling inhomogeneity within the visual system. Manuscript submitted for publication.

GARCÍA-PÉrez, M. A. (1988). Space-variant visual processing: Spatially limited visual channels. Spatial Vision, 3, 129-142.

GarCíA-PÉrez, M. A. (1989). Visual inhomogeneity and reference frames. Bulletin of the Psychonomic Society, 27, 21-24.

GinsBurG, A. P. (1978). Visual information processing based on spatial filters constrained by biological data. (Doctoral dissertation AMRI-TR-78-129). Aerospace Medical Research Laboratory, WrightPatterson Air Force Base, Wright-Patterson, Ohio.

Goolkasian, P. (1987). Ambiguous figures: Role of context and critical features. Journal of General Psychology, 114, 217-228.

HiLL, W. E. (1915). My wife and my mother-in-law [Cartoon]. Puck. Hochstein, S., \& SPITZER, H. (1984). Zero-crossing detectors in primary visual cortex? Biological Cybermerics, 51, 195-199.

Kawabata, N. (1986). Attention and depth perception. Perception, 15, 563-572.

Kawamoto, A. H., \& ANDERson, J. A. (1985). A neural network model of multistable perception. Acta Psychologica, 59, 35-65.
Kienker, P. K., Sejnowski, T. J., Hinton, G. E., Schumacher, L. E. (1986). Separating figure from ground with a parallel network. Perception, 15, 197-216.

KöHLER, W., WALLACH, H. (1944). Figural aftereffects, an investigation of visual processes. Proceedings of the American Philosophical Society, 88, 269-357.

LIEBERT, R. M., BURK, B. (1985). Voluntary control of reversible figures. Perceprual \& Motor Skills, 61, 1307-1310.

Long, G. M., Toppino, T. C., Kostenbauder, J. F. (1983). As the cube turns: Evidence for two processes in the perception of a dynamic reversible figure. Perception \& Psychophysics, 34, 29-38.

MARR, D. (1982). Vision. San Francisco: W. H. Freeman.

Poston, T., \& Stewart, I. (1978). Nonlinear modeling of multistable perception. Behavioural Science, 23, 318-334.

RICHTER, J., \& UlLMAN, S. (1986). Non-linearities in cortical simple cells and the possible detection of zero crossings. Biological Cybernetics, 53, 195-202.

Robinson, J. O. (1972). The psychology of visual illusion. London: Hutchinson.

SHAFER, M. (1976). A catastrophe theory model for ambiguous picture perception (Social Sciences Working Paper No. 110a). Irvine: University of Califomia, School of Social Sciences.

TSAL, Y., KOLBET, L. (1985). Disambiguating ambiguous figures by selective attention. Quarterly Joumal of Experimental Psychology, 37A, 25-37.

WiLton, R. N. (1979). The recency effect in the perception of ambiguous figures. Perception, 14, 53-61.

(Manuscript received August 30, 1988; revision accepted for publication March 27, 1989.) 